\section{Overtube and over-the-wire, through-the-scope balloon-assisted ERCP in a patient with complex hypopharyngeal stenosis after neck surgery}

A 64-year-old man with T2N0 squamous cell carcinoma of the tongue base underwent treatment with radiation, followed by glossolaryngectomy, bilateral neck dis- section, and placement of an anterolateral thigh flap graft. After 1 year, he presented with acute cholangitis (abdominal pain, jaundice, fever, chills, and hypotension), making emergency endoscopic retrograde cholangiopancreatography (ERCP) mandatory. A computed tomographic scan of the neck showed hypopharyngeal stenosis and neck swelling as a result of the previous surgery ( $\bullet$ Fig.1a, $\bullet$ Video 1 ). At another institution, emergency ERCP had failed, and the patient was transferred to our institution for further treatment.

On standard esophagogastroduodenoscopy, the changes due to the prior com-
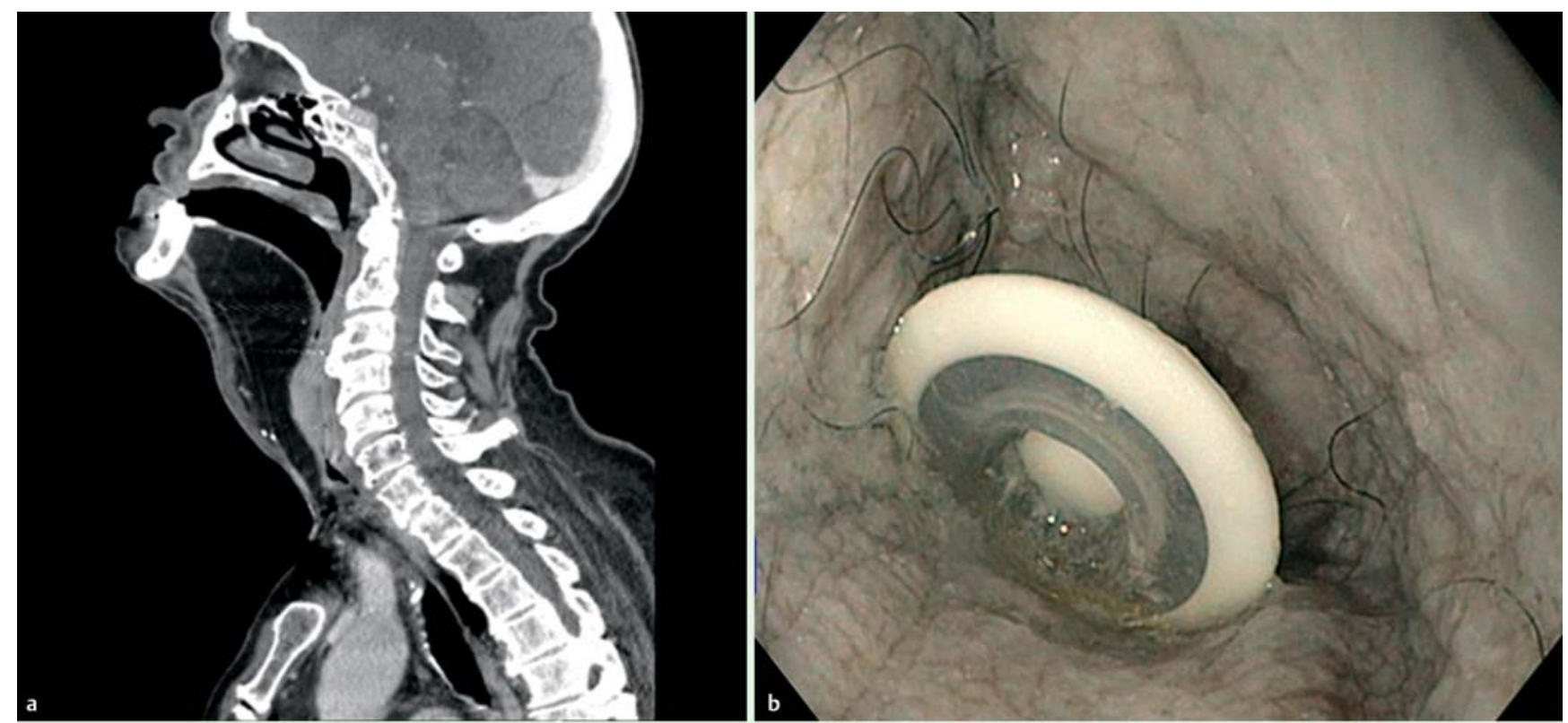

4
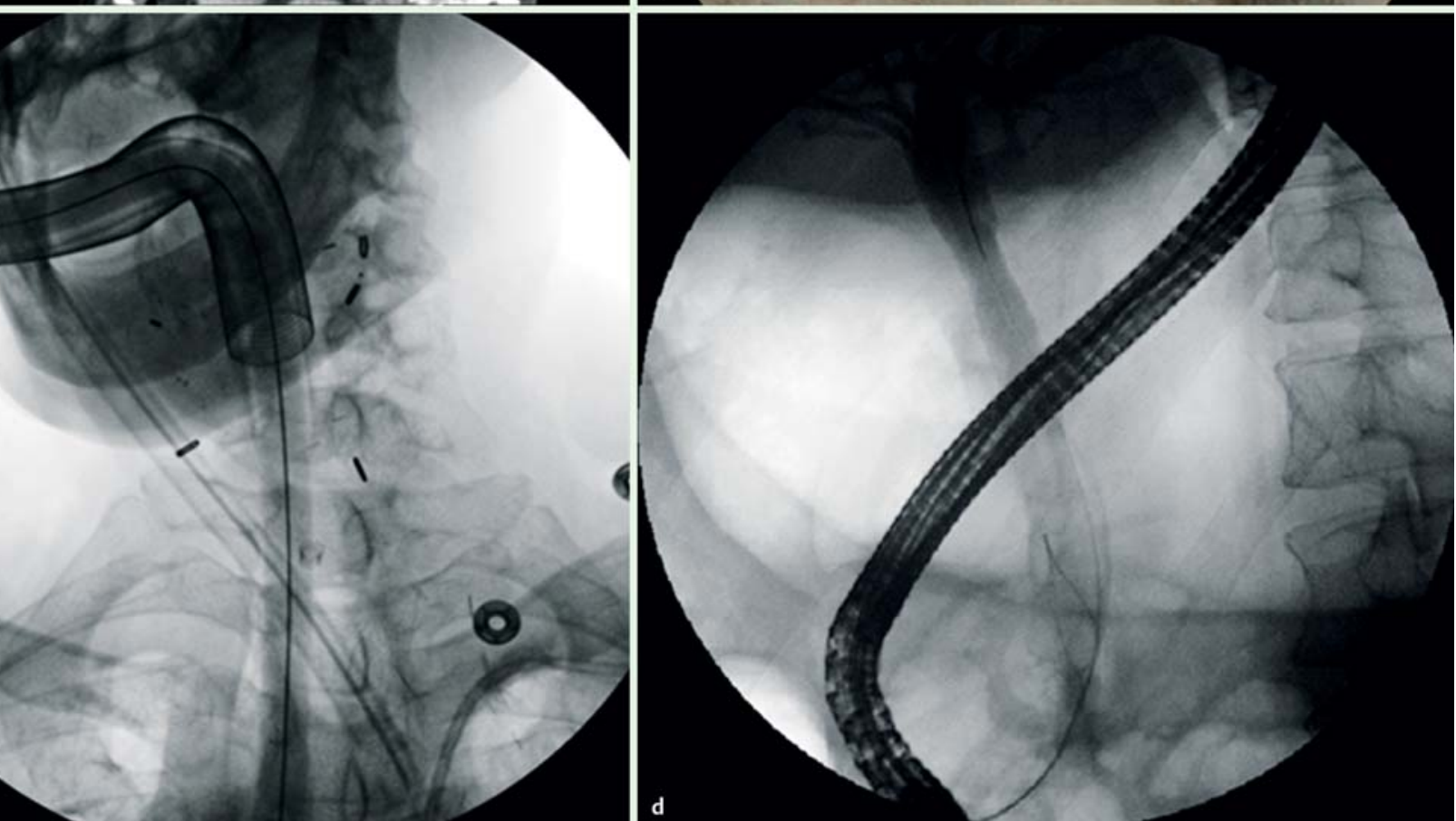

Fig 1 a Computed tomographic scan showing hypopharyngeal stenosis and neck swelling in a 64-year-old man as a result of previous surgery for squamous cell carcinoma of the tongue base. $\mathbf{b}$ Stenosis between the hypopharynx and upper esophageal sphincter induced by radiation fibrosis and the presence of a flap graft from the thigh. Note the hairy skin graft and the voice valve. $\mathbf{c}$ The presence of an overtube, which functioned as a giant working channel, allowed passage of the duodenoscope. $\mathbf{d}$ Adequate scope position for endoscopic retrograde cholangiopancreatography (ERCP), which was then conducted in standard fashion. Sphincterotomy with stone removal was accomplished successfully. 
plex radiation and surgery of the hypopharynx were evident. The hypopharynx was stenotic and fibrosed. A hairy skin graft and a voice valve were present $(\bullet$ Fig.1b). Although the gastroscope could be passed into the stomach without major problems, it was impossible to advance a side-viewing duodenoscope. Therefore, using a standard gastroscope, we carefully placed an overtube (Guardus overtube-esophageal; inner diameter $16.7 \mathrm{~mm}$, outer diameter $19.9 \mathrm{~mm}$; US Endoscopy, Mentor, Ohio, USA) ( $\bullet$ Fig. 1 c). The inner overtube was removed, and the outer overtube was left in situ. The presence of the overtube permitted passage of the side-viewing ERCP scope, preventing damage and laceration of the stenotic graft and voice apparatus ( $\bullet$ Video 1 ).

A wire-guided, through-the-scope balloon (Hercules, 10-11-12 mm; Cook Medical, Winston-Salem, North Carolina, USA) was used to dilate the stenosis gently and serve as a guide and pathfinder for the scope. Under combined fluoroscopic and endoscopic visualization, the scope was gently pushed behind the inflated balloon and advanced beyond the stenosis, and the ERCP was then conducted in standard fashion ( $\bullet$ Fig. 1 d). A sphincterotomy with stone removal was accomplished successfully, the patient's condition improved rapidly, and he was discharged in stable condition 2 days later.

This case demonstrates the life-saving use of an overtube to perform emergency ERCP in the setting of a hypopharyngealupper esophageal stricture and cholangi-

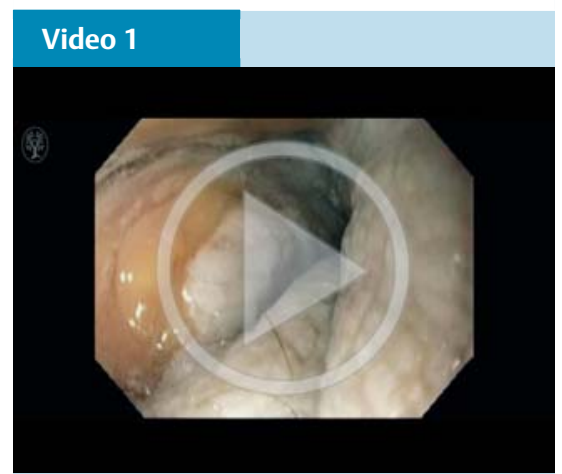

Overtube and over-the-wire, through-thescope balloon-assisted endoscopic retrograde cholangiopancreatography in a patient with complex hypopharyngeal stenosis after neck surgery.

tis. To the best of our knowledge, we are not aware of any report describing a similar technique of overtube and over-thewire, through-the-scope balloon-assisted ERCP. The large-diameter overtube served as a "giant working channel," permitting passage of a side-viewing duodenoscope. The overtube served two purposes: (i) bridging the stenosis and (ii) straightening the oropharyngeal-esophageal channel, which allowed the unrestricted and safe passage of a duodenoscope. The balloon served as a guide, dilator, and "pathfinder." Therefore, this life-saving technique of overtube and over-the-wire, throughthe-scope balloon-assisted ERCP should be added to the armamentarium of the therapeutic endoscopist.
Endoscopy_UCTN_Code_TTT_1AR_2AH

Competing interests: None

\section{Sandhya Mudumbi, Esraa Esmat Mohamed, Paul T. Kröner, Lucia C. Fry, Kondal R. Kyanam Kabir Baig, Klaus Mönkemüller}

Basil I. Hirschowitz Endoscopic Center of Excellence, Division of Gastroenterology and Hepatology, University of Alabama at Birmingham, Birmingham, Alabama, USA

\section{Bibliography}

Dol http://dx.doi.org/

10.1055/s-0034-1392871

Endoscopy 2015; 47: E542-E543

(c) Georg Thieme Verlag KG

Stuttgart · New York

ISSN 0013-726X

\section{Corresponding author}

\section{Klaus Mönkemüller, MD, PhD, FASGE}

Division of Gastroenterology and Hepatology Basil I. Hirschowitz Endoscopic

Center of Excellence

Endoscopy Unit, JT 664

619 19th Street $S$

Birmingham, AL 35249

USA

Fax: +1-205-297-9411

klaus1@uab.edu 